\title{
Resultados iniciales con bioglue en la prevención de fístulas urinarias en cirugía renal conservadora laparoscópica
}

\author{
Núñez Mora C, García Mediero JMª . \\ Servicio de Urología. Centro Oncológico MD Anderson Internacional España. Madrid.
}

Actas Urol Esp. 2008;32(3):316-319

\section{RESUMEN}

RESULTADOS INICIALES CON BIOGLUE EN LA PREVENCIÓN DE FÍSTULAS URINARIAS EN CIRUGÍA RENAL CONSERVADORA LAPAROSCÓPICA

Introducción: La aparición de una fístula urinaria constituye una complicación de la cirugía renal conservadora de parénquima (CRCP), sobre todo en tumores de localización central o hiliar y en aquellos mayores de $4 \mathrm{~cm}$ de diámetro. Presentamos nuestra experiencia inicial en su prevención utilizando BioGlue tras la realización de CRCP laparoscópica en las que se produce apertura de la vía urinaria.

Pacientes y métodos: Entre septiembre de 2005 y febrero de 2006 se han realizado 5 CRC laparoscópicas en las que ha sido necesario proceder a la apertura de la vía urinaria. En todos los casos se realizó cateterismo ureteral previo. Mediante abordaje laparoscópico transperitoneal se realizó clampaje arterial selectivo, tumorectomía o heminefrectomía, sutura de la vía urinaria comprobando estanqueidad, aproximación de los bordes de la incisión renal y recubrimiento de la misma con adhesivo tisular compuesto por glutaraldehido y albúmina (BioGlue). El seguimiento medio fue de 8,2 meses (rango 6-12 meses).

Resultados: La duración media de la cirugía fue de 138 minutos (rango 105-180), con un tiempo medio de isquemia de 45 minutos (rango 35-60). El catéter ureteral se retiró en todos los casos entre las 24 y 48 horas postoperatorias. La estancia media fue de 3,8 días (rango 3-5). Como complicaciones se produjo un defecto del clampaje arterial por el torniquete de Rummel que requirió transfusión intraoperatoria y una fístula arterio-calicial al décimo día postoperatorio que requirió reingreso y embolización selectiva. No se produjeron fístulas urinarias ni urinomas. En la TAC al mes de la cirugía se apreció en un caso una fístula urinaria contenida por el Bio-glue. Dicha fístula había desaparecido en el control a los 3 meses de la cirugía.

Conclusiones: En la CRCP laparoscópica de masas que interesen la vía renal, la realización de un cateterismo ureteral previo, la sutura de la vía por separado junto con la aplicación de BioGlue permite reducir la posibilidad de una fístula urinaria.

Palabras clave: Pegamento quirúrgico. Tumorectomía renal laparoscópica. Cáncer renal.

\begin{abstract}
PREVENTING URINARY FISTULAS IN LAPAROSCOPIC RENAL CONSERVATIVE PARENCHIMA SURGERY WITH PURIFIED BOVINE SERUM ALBUMIN AND GLUTARALDEHYDE (BIOGLUE ${ }^{\circledR}$ ). INITIAL OUTCOMES

Introduction: Urinary fistulas remain an important conservative renal parenchyma surgery (CRPS) complication, especially in central or hiliar tumours and bigger than $4 \mathrm{~cm}$. Herein we present our initial experience preventing fistulae with bioglue ${ }^{\circledR}$ (Criolife Inc GA, USA) on laparoscopic CRPS in which urinary tract was opened.

Patients and methods: We performed 5 laparoscopic CRPS between September 2005 and February 2006 in which urinary tract was necessarily opened. Previous uretheral catheter, transperitoneal approach, selective arterial control, tumorectomy or heminephrectomy, suturing urinary tract and renal parenchyma and bioglue ${ }^{\circledR}$ administration was performed.

Results: Median follow up time was 8.2 months (6-12 months). Median surgery time was 138 minutes (105-180 minutes) with a median ischemia time of 45 minutes (35-60). Uretheral catheter was removed before second post-op day in all cases. Average discharged day was 3.8 (3-5 days). One patient required intraoperative transfusion due to breaking Rummel tourniquet and one arterio- calyceal fistula on tenth day pos-op that required selective embolization remained the worst complication. Neither urinary fistulas nor urinomas were reported.

Conclusions: Uretheral cathetesim, suturing urinary tract and parenchyma in an independent fashion and applying some kind of surgical adhesive such as bioglue ${ }^{\circledR}$ seems to reduce the urinary fistulae risk in laparoscopic CRPS.
\end{abstract}


$\mathrm{L}^{\mathrm{a}}$ a CRC está ampliamente aceptada en tumores renales inferiores a $4 \mathrm{~cm}^{1,2}$ e incluso algunos estudios han demostrado una eficacia similar de la CRCP a la de la nefrectomía radical en tumores de hasta $7 \mathrm{~cm}^{1,3}$. El abordaje laparoscópico de estos tumores, superiores a $4 \mathrm{~cm}$, así como de aquellos de localización central o hiliar conlleva una mayor necesidad de apertura de la vía urinaria, con el consiguiente riesgo de desarrollar fístulas urinarias ${ }^{4,5}$. Presentamos nuestra experiencia inicial en la prevención de fístulas urinarias en la CRCP laparoscópica mediante la utilización de un adhesivo tisular compuesto por glutaraldehido y albúmina (BioGlue, Cryolife).

\section{PACIENTES Y MÉTODOS}

Entre julio de 2005 y febrero de 2006 hemos realizado 5 CRCP laparoscópicas en las que, bien por el tamaño tumoral, o bien por la localización del mismo fue necesario proceder a la apertura de la vía urinaria (Figs. 1 y 2).

Las características de los pacientes y de los tumores quedan recogidas en la Tabla 1 .

En todos los casos, ante la sospecha de afectación de la vía en la TAC o RMN, se realizó un cateterismo ureteral previo al abordaje laparoscópico. Éste se realizó mediante acceso transperitoneal realizando los siguientes pasos:

1. Liberación renal y exposición del tumor.

2. Disección hiliar y clampaje selectivo de arteria renal-en tres casos con torniquete de Rummel laparoscópico y en otros dos con bulldogs laparoscópicos.

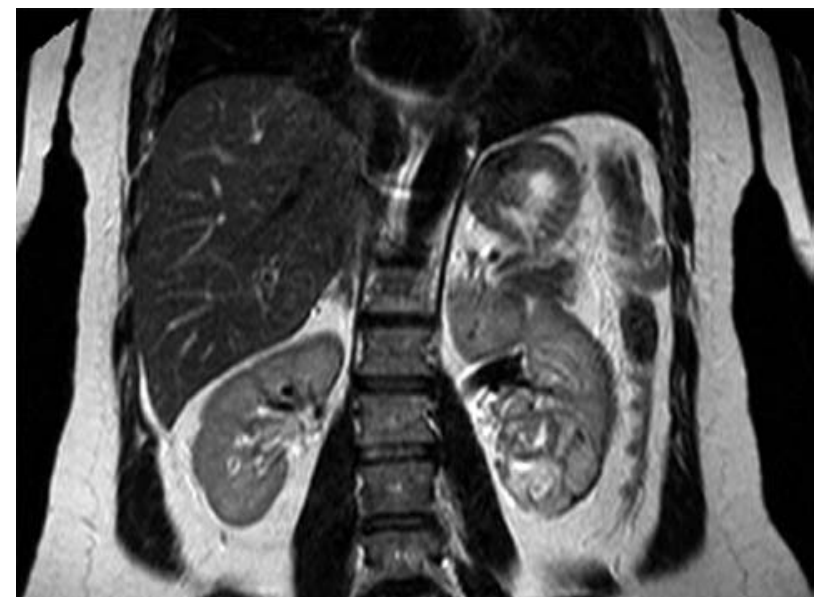

FIGURA 1. Caso número 5. RMN mostrando un tumor de 5,6 cm en el polo inferior renal en intimo contacto con el tracto urinario. Se realizó heminefrectomía laparoscópica.

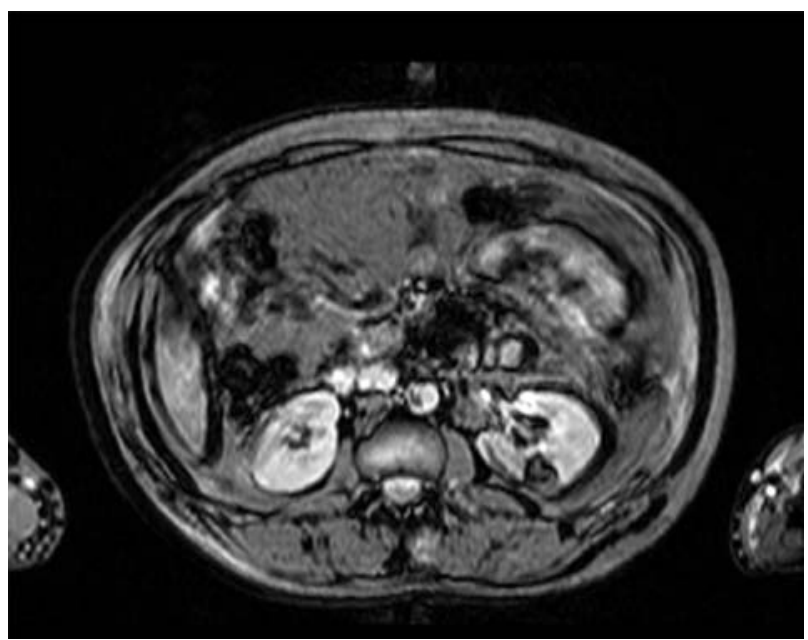

FIGURA 2. Paciente número 4. Tumor renal izquierdo central próximo a la pelvis renal. Este paciente desarrolló una fistula al décimo día postoperatorio que se resolvió con embolización selectiva.

3. Tumorectomía con corte frío.

4. Biopsia intraoperatoria del lecho tumoral y embolsamiento de la pieza.

5. Sutura continua de la vía urinaria comprobando su estanqueidad mediante la infusión de suero por el catéter ureteral.

6. Aproximación de los bordes de la incisión renal mediante puntos sueltos.

7. Cobertura de la herida renal con dos capas de BioGlue.

La retirada del catéter ureteral se realizó tras comprobar escaso débito por el drenaje quirúrgico el cual se retiró posteriormente.

Todos los pacientes fueron sometidos a exploración con TAC al mes de la cirugía.

El seguimiento medio fue de 8,2 meses, con un mínimo de 6 meses.

\section{RESULTADOS}

La duración media de la cirugía fue de 138 minutos (rango 105-180), con un tiempo medio de isquemia de 45 minutos (rango 35-60).

En un caso la hemostasia proporcionada por el torniquete de Rummel laparoscópico no fue adecuada, requiriendo su recolocación y necesitando transfusión intraoperatoria. Ningún otro paciente requirió transfusión sanguínea peroperatoria.

Las biopsias intraoperatorias del lecho renal fueron negativas en todos los casos.

El estudio histopatológico de los tumores reveló la presencia de carcinoma renal de células claras en 4 casos y carcinoma quístico multilocular en otro. 
Tabla 1. Características de los pacientes. Todos los tumores se encontraban en íntimo contacto con la vía urinaria

\begin{tabular}{lcclclcc}
\hline $\begin{array}{l}\text { Número } \\
\text { Caso }\end{array}$ & Sexo & Edad & $\begin{array}{l}\text { Antecedentes } \\
\text { quirúrgicos }\end{array}$ & Lado & Localización & $\begin{array}{c}\text { Tamaño } \\
\text { (CT, RMN) }\end{array}$ & Tipo de cirugía \\
\hline 1 & V & 52 & $\begin{array}{l}\text { Colecistectomía } \\
\text { laparoscópica } \\
\text { Colecistectomía } \\
\text { Laparoscópica + Nissen }\end{array}$ & R & Polo Superior & $5,4 \mathrm{~cm}$ & Tumorectomía \\
2 & V & 59 & L & Valva Anterior, & $5,2 \mathrm{~cm}$ & Tumorectomía \\
3 & V & 52 & 52 & L & $\begin{array}{l}\text { Vilium } \\
\text { Valva Posterior, }\end{array}$ & $3 \mathrm{~cm}$ & Tumorectomía \\
4 & V & & L & Polo inferior & $5,6 \mathrm{~cm}$ & Heminefrectomía \\
5 & M & 40 & & & &
\end{tabular}

La tolerancia oral se inició, en todos los casos, a las 12 horas de la intervención. El catéter ureteral se retiró a las 24 horas en 3 casos y a las 48 horas en los otros dos. En éstos últimos el catéter se mantuvo por alto débito del drenaje en las primeras 24 horas (?200cc) y se retiró tras demostrar en el análisis bioquímico que el líquido drenado tenía características plasmáticas. Los drenajes se retiraron 24 horas después de la retirada de los catéteres ureterales, tras comprobar que presentaban un débito escaso de características plasmáticas.

La estancia media fue de 3,8 días (rango 3-5 días).

Uno de los pacientes presentó a los 10 días de la cirugía un cuadro de fístula arterio-calicial con hematuria anemizante que requirió embolización selectiva.

En la TAC realizada al mes de la cirugía no se apreció en ningún caso la presencia de urinomas o colecciones perirrenales significativas. En el caso número 1 se apreciaba una fístula urinaria contenida por la capa de BioGlue (Fig. 3), que evolucionó sin formación de urinoma hasta su desaparición en el control a los 3 meses de la cirugía (Fig. 4).

A los 6 meses postoperatorios no se detectó deterioro de la función renal en ninguno de los pacientes.

\section{DISCUSIÓN}

La aparición de fístulas urinarias tras CRCP es muy variable en la literatura, aunque en la mayoría de las series oscila entre el 0,6\% y el 10,1\% de los $\operatorname{casos}^{4,6-10}$. Esta incidencia, relativamente baja, se explica porque muchas de las series publicadas incluyen tumores con un tamaño medio inferior a $3,5 \mathrm{~cm}^{4,5,9}$, por lo que salvo en tumores de localización hiliar o central ${ }^{4,5}$ (Fig. 5) no se suele producir apertura de la vía urinaria. A pesar de esto, las fístulas urinarias constituyen la complicación más fre-

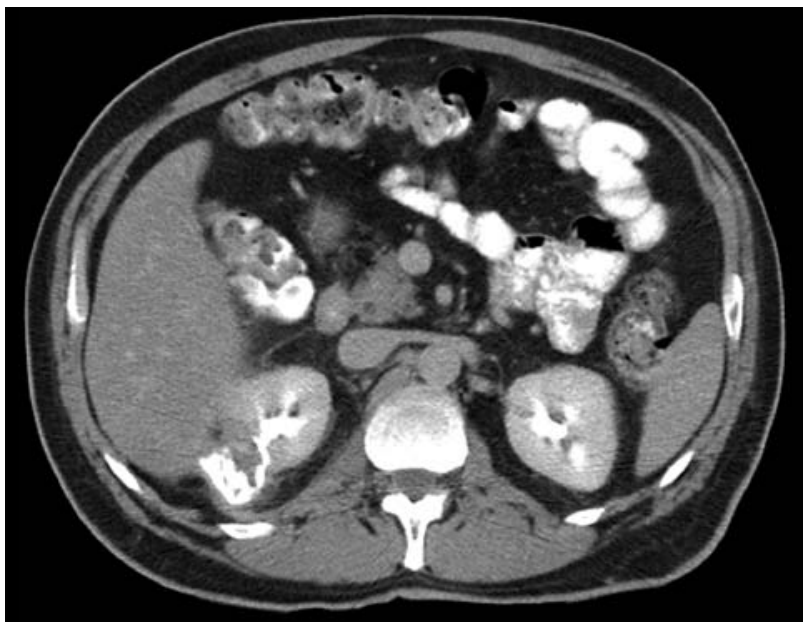

FIGURA 3. El paciente número 1 desarrolló una fistula de forma asintomática contenida por el pegamento quirúrgico bioglue $\odot$.

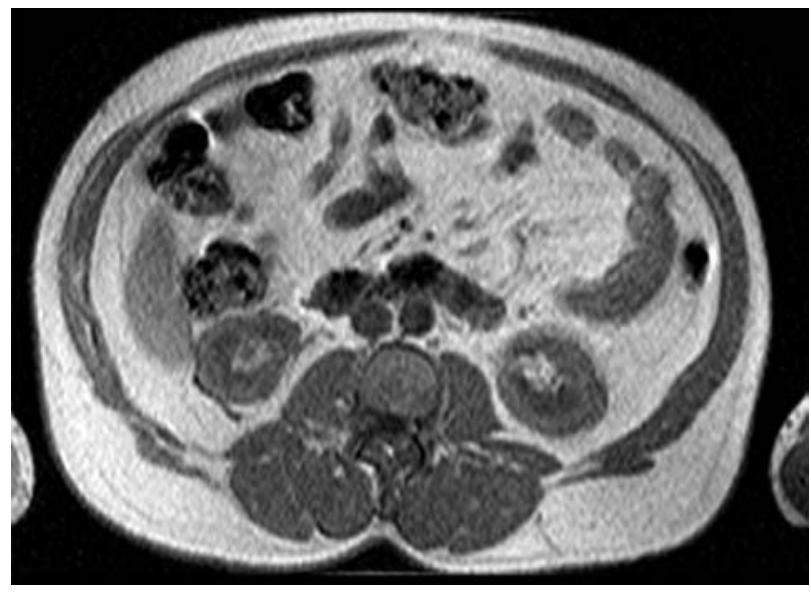

FIGURA 4. Fistula contenida del paciente num. 1 que evolucionó hasta su completa desaparición en un CT al tercer mes.

cuente de la CRCP en el subgrupo de grandes tumores endofíticos o hiliares en localización ${ }^{7,8,10}$ y ocasionan una prolongación de la estancia hospitalaria y de la tasa de reintervenciones ${ }^{8,10}$. 


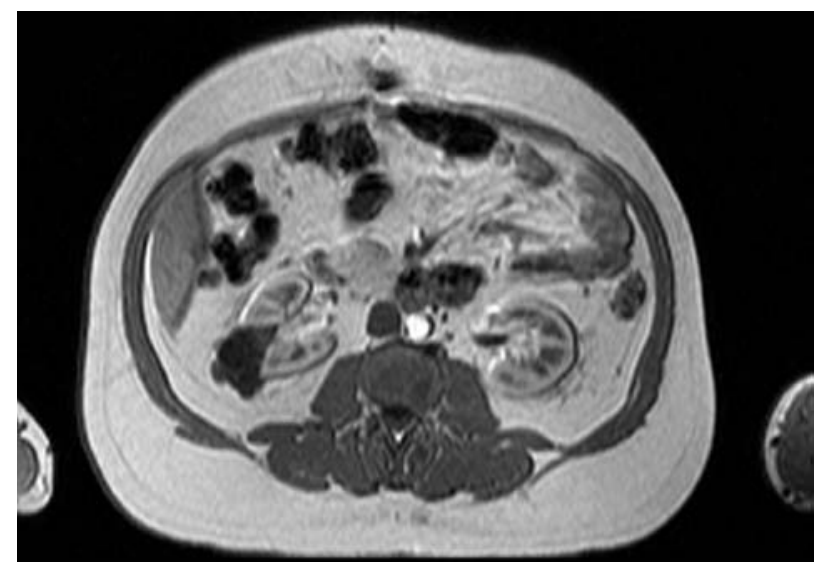

FIGURA 5. Paciente num. 2. Carcinoma renal quistico multilocular. Nótese su íntimo contacto con el tracto urinario.

En CRCP laparoscópica se ha llegado a publicar una tasa del 50\% de fístulas urinarias en el caso de tumores hiliares ${ }^{5}$, por lo que es previsible que según aumente el tamaño de los tumores operados aumentará paralelamente la necesidad de apertura de la vía urinaria y con ella la tasa de fístulas urinarias. Para prevenir estas últimas consideramos necesario realizar un cateterismo ureteral previo en aquellos tumores que se encuentren cercanos a la vía urinaria (Figs. 1 y 2) con el fin de infundir suero a su través, tras la tumorectomía, y así detectar y suturar las posibles aperturas de la vía urinaria.

La aplicación de sustancias que mejoren la hemostasia en la CRCP laparoscópica está ampliamente difundida, siendo probablemente el Floseal el más utilizado ${ }^{11-14}$, pero respecto a la impermeabilización de la vía urinaria los estudios realizados con Floseal presenta hasta un $25 \%$ de fístulas ${ }^{15}$. Respecto al BioGlue, tras su coagulación toma una consistencia poco elástica, impermeable, semejante a la silicona. Por esta razón su eficacia como agente impermeabilizante ha sido baja cuando se ha utilizado en anastomosis dinámicas como las uretrovesicales en modelos animales ${ }^{16}$. Sin embargo, su eficacia en el control de la hemostasia ha sido demostrada por Hidas et al. ${ }^{17} \mathrm{y}$, como se aprecia en nuestro caso 1 , es capaz de contener una fístula urinaria, impidiendo así la formación de un urinoma perirrenal.

Aunque nuestra serie inicial es corta consideramos que la aplicación de BioGlue tras la sutura de la vía urinaria en la CRCP laparoscópica puede reducir la aparición de fístulas urinarias en este subgrupo de pacientes con tumores grandes, endofíticios y/o hiliares.

\section{CONCLUSIONES}

La realización de CRCP laparoscópica en tumores mayores de $4 \mathrm{~cm}$ o de localización hiliar o central conlleva una elevada tasa de apertura de vía urinaria con el consiguiente riesgo de fístulas urinarias postoperatorias. En estos casos la sutura de la vía urinaria junto con la aplicación de BioGlue permite reducir este riesgo.

\section{REFERENCIAS}

1. Patard JJ, Shvarts O, Lam JS, Pantuck AJ, Kim HL, Ficarra V, et al: Safety and efficacy of partial nephrectomy for all T1 tumors based on an international multicenter experience. J Urol. 2004;171(6 Pt 1):2182-2185.

2. Fergany AF, Hafez KS, Novick AC. Long.term results of nephron sparing surgery for localized renal cell carcinoma: 10-year followup. J Urol. 2000; 163(2):442-445.

3. Leibovich BC, Blute ML, Cheville JC, Lohse CM, Weaver AL, Zincke H. Nephron sparing surgery for appropriately selected renal cell carcinoma between 4 and $7 \mathrm{~cm}$ results outcome similar to radical nephrectomy. $J$ Urol. 2004;171(3):1066-1070.

4. Drachenberg DE, Mena OJ, Choyke PL, Linehan WM, Walther MM. Parenchymal sparing surgery for central renal tumors in patients with hereditary renal cancers. J Urol. 2004;172(1):49-53.

5. Reisiger K, Venkatesh R, Figenshau RS, Bae KT, Landman J. Complex laparoscopic partial nephrectomy for renal hilar tumors. Urology. 2005;65 (5):888-891.

6. Thompson RH, Leibovich BC, Lohse CM, Zincke H, Blute ML. Complications of contemporary open nephron sparing surgery: a single institution experience. J Urol. 2005; 174(3):855-858.

7. Uzzo RG, Novick AC. Nephron sparing surgery for renal tumors: indications, techniques and outcomes. J Urol. 2001;166(1):16-18.

8. Stephenson AJ, Hakimi AA, Snyder ME, Russo P. Complications of radical and partial nephrectomy in a large contemporary cohort. J Urol. 2004;171 (1): $130-134$.

9. Abukora F, Nambirajan T, Albqami N, Leeb K, Jeschke S, Gschwendtner M, et al. Laparoscopic nephron sparing surgery: evolution in a decade. Eur Urol. 2005;47(4):488-493.

10. Pasticier G, Timsit MO, Badet L, De La Torre Abril L, Halila M, Fassi Fehri $\mathrm{H}$, et al. Nephron-sparing surgery for renal cell carcinoma: detailed analysis of complications over a 15-year period. Eur Urol. 2006;49(3):485-490.

11. User HM, Nadler RB. Applications of FloSeal in nephron-sparing surgery. Urology. 2003;62(2):342-343.

12. Richter F, Scnorr D, Deger S, et al: Improvement of hemostasis in open and laparoscopically performed partial nephrectomy using a gelatine matrixthrombin tissue sealant (FloSeal). Urology 61: 73-77, 2003.

13. Richter F, Tüllmann ME, Türk I, et al: Verbesserung del Hämostase bei laparoskopischen und offenen Teilnephrektomien durch Gelatine-ThrombinMatrix (FloSeal). Urologe(A) 3: 338-346, 2003.

14. Guazzoni G. Tissue sealants in laparoscopic conservative renal surgery. Eur Urol. 2006;50(4):650-652.

15. L'Esperance JO, Sung JC, Marguet CG, Maloney ME, Springhart WP, Preminger GM, et al: Controlled survival study of the effects of Tisseel or a combination of FloSeal and Tisseel on major vascular injury and major collecting-system injury during partial nephrectomy in a porcine model. J Endourol. 2005; 19(9):1114-1121.

16. Hruby G, Marruffo F, Durak E, Collins S, Herron A, Landman J. Comparison of BioGlue reinforced and standard running sutured vesicourethral anastomoses. Urology. 2006;68(6): 1355-1359.

17. Hidas G, Kastin A, Mullerad M, Shental J, Moskovitz B, Nativ O. Sutureless nephron-sparing surgery: use of albumin glutaraldehyde tissue adhesive (BioGlue). Urology. 2006;67(4): 697-700.

Correspondencia autor: Dr. J.M ${ }^{\mathrm{a}}$ García Mediero

Servicio de Urología

Centro Oncológico MD Anderson Internacional España

Arturo Soria, 270 - 28033 Madrid. Tel.: 917878600

E-mail autor: garciamediero@hotmail.com

Información artículo: Cáncer renal

Trabajo recibido: marzo 2007

Trabajo aceptado: septiembre 2007 